\title{
Screening of ruptured plaques in patients with coronary artery disease by intravascular ultrasound
}

J Ge, F Chirillo, J Schwedtmann, G Görge, M Haude, D Baumgart, V Shah, $\mathrm{C}$ von Birgelen, S Sack, H Boudoulas, R Erbel

\begin{abstract}
Aim-To visualise the characteristics of ruptured plaques by intravascular ultrasound (IVUS) and to correlate plaque characteristics with clinical symptoms to establish a quantitative index of plaque vulnerability.

Methods-144 consecutive patients with angina were examined using IVUS. Ruptured plaques, characterised by a plaque cavity and a tear on the thin fibrous cap, were identified in 31 patients (group A), of whom $23(74 \%)$ presented with unstable angina. Plaque rupture was confirmed by injecting contrast medium filling the plaque cavity during IVUS examination. Of the patients without plaque rupture (group $B, n=108)$, only $19(18 \%)$ had unstable angina.
\end{abstract}

Results-No significant differences were found between groups $A$ and $B$ in relation to plaque and vessel area $(p>0.05)$. Mean (SD) per cent stenosis in group $A$ was less than in group $B$, at $56.2(16.5) \% \quad v 67.9$ $(13.4) \% ; p<0.001$. Area of the emptied plaque cavity in group A $\left(4.1(3.2) \mathrm{mm}^{2}\right)$ was larger than the echolucent zone in group B $\left(1.32(0.79) \mathrm{mm}^{2}\right)(p<0.001)$. The plaque cavity to plaque ratio in group $A$ (38.5 (17.1)\%) was larger than the echolucent area to plaque ratio in group $B(11.2$ $(8.9) \%)(p<0.001)$. The thickness of the fibrous cap in group $A$ was less than in group $B$, at $0.47(0.20) \mathrm{mm} v 0.96(0.94)$ mm; p $<0.001$.

Conclusions-Plaques seem to be prone to rupture when the echolucent area is larger than $4.1(3.2) \mathrm{mm}^{2}$, when the echolucent area to plaque ratio is greater than 38.5 $(\mathbf{1 7 . 1}) \%$, and when the fibrous cap is thinner than $0.7 \mathrm{~mm}$. IVUS can identify plaque rupture and vulnerable plaques. This may influence patient management and treatment.

(Heart 1999;81:621-627)

Cardiology, Regional Hospital Treviso, Italy F Chirillo

Division of Cardiology, Ohio State University,

Ohio, USA

$\mathrm{H}$ Boudoulas

Correspondence to: Dr Ge.

Accepted for publication 15 December 1998

Plaque rupture and the subsequent thrombus formation are major events which lead to complex lesions and contribute to the pathogenesis of acute coronary syndromes such as unstable angina, myocardial infarction, and sudden death. ${ }^{1-5}$ After plaque rupture, the atheroma can be washed out and an open cavity remains. ${ }^{156}$ Coronary angiography, which provides a silhouette of the vessel lumen, can only indirectly suggest plaque rupture in the form of a filling defect caused by intraluminal thrombus or a contrast filled crater from deep ulceration. $^{78}$

Intravascular ultrasound (IVUS) offers a new method of for visualising the coronary artery wall, plaque morphology, and plaque composition, ${ }^{9-14}$ and as such has the potential to identify plaque disruption, as reported in a preliminary study. ${ }^{15}$ The purposes of the present study were first, to visualise the characteristics of ruptured plaques and second, to correlate the plaque characteristics with clinical symptoms and to establish a quantitative index of plaque vulnerability with IVUS.

\section{Methods}

PATIENTS

In our cardiovascular catheterisation laboratory, we used IVUS after coronary angiography to examine 144 consecutive patients (aged from 35 to 75 years) who present with angina associated with ischaemic ECG changes on exercise. To evaluate unstable patients (within two weeks), we used the clinical classification proposed by Braunwald. ${ }^{16}$ All patients provided written informed consent for IVUS examination. Patients with acute myocardial infarction and in an unstable situation, such as cardiogenic shock, who were not suitable for IVUS, were not included.

\section{THE IVUS DEVICE}

The IVUS imaging system used in this study has been described previously in detail. ${ }^{67} \mathrm{~A}$ $3.5 \mathrm{~F}$ imaging catheter with a single 20 or 30 $\mathrm{MHz}$ element transducer at the tip (Sonicath, Boston Scientific Corporation, Watertown, Massachusetts, USA) was used. The transducer is mechanically rotated within the catheter at 600 to $900 \mathrm{rpm}$ to provide cross sectional images using an ultrasound diagnostic imaging console (Sonos 1000; HewlettPackard, Andover, Massachusetts, USA). The images were stored on $1 / 2$ inch s-VHS videotape for offline analysis. An ECG was recorded simultaneously.

\section{PROCEDURE}

Each IVUS study was carried out according to a standard procedure ${ }^{67}$ After diagnostic coronary angiography, a bolus of heparin (3000 IU) was given by intracoronary injection. An $8 \mathrm{~F}$ giant lumen guiding catheter (Medtronic, 

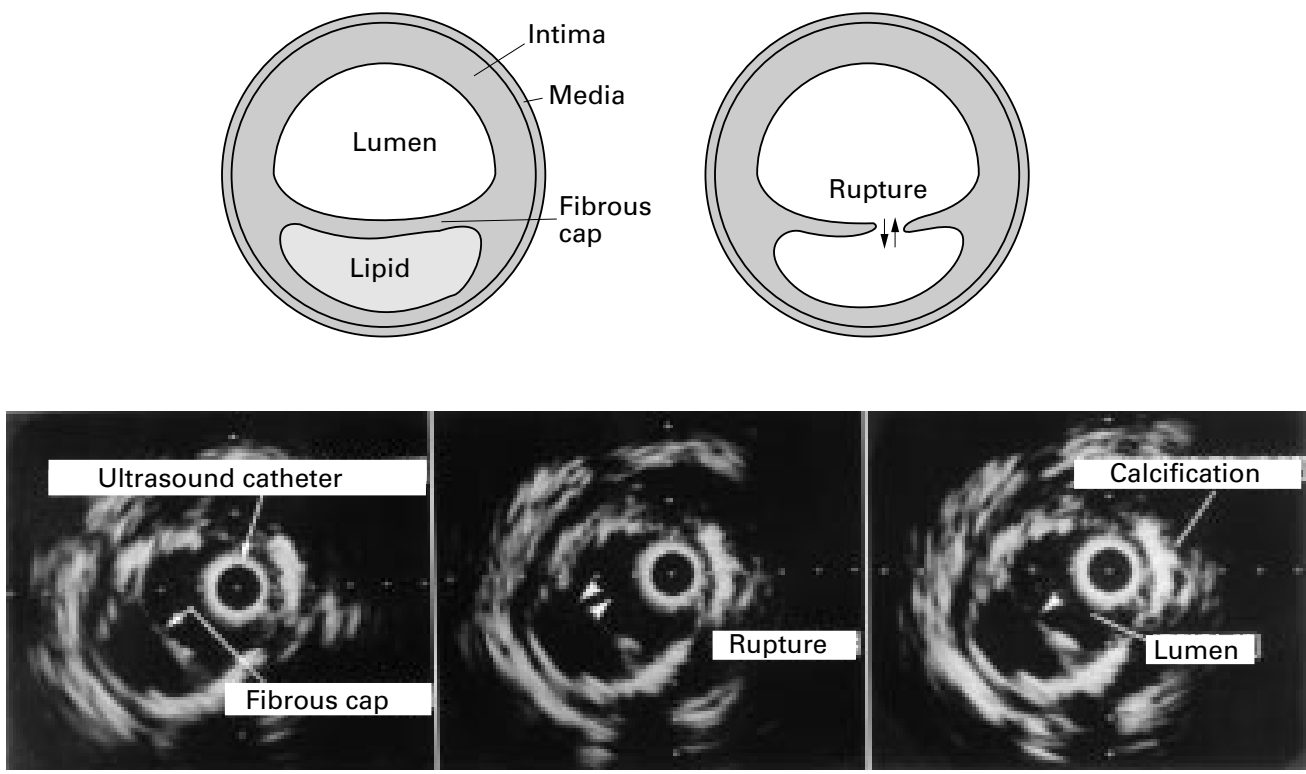

Figure 1 Schematic drawing (upper panel) and IVUS images of plaque rupture (lower panel). The ruptured plaque is characterised by a narrow tear in a thin fibrous cap and an emptied echolucent zone (type I).

Denvers, Massachusetts; or Cordis, Miami, Florida, USA) was positioned into the coronary ostium of the culprit vessel. A 0.014 inch $(0.36 \mathrm{~mm})$ guide wire was inserted into the coronary artery. The IVUS side saddle catheter was guided in a mono-rail fashion into the left coronary artery. The catheter was advanced as far as possible into the target coronary artery. Serial cross sectional images of the vessel were obtained by slowly pulling back the IVUS catheter, with stop frames at 2 to $3 \mathrm{~mm}$ intervals. The position of the IVUS probe was documented on $x$ ray film at every stop frame in order to obtain precise matching between the numbered IVUS images and the angiograms. Contrast medium was injected through the guiding catheter in order to enhance the IVUS imaging.

IVUS IMAGE ANALYSIS

The IVUS images were reviewed online by the operator and offline from the tapes by an independent observer. Plaque rupture (fig 1) was diagnosed by visualising an echolucent zone (cavity) within a plaque, separated from the lumen by a thin echoreflectant structure representing the fibrous cap (type I). Communication between the lumen of the vessel and plaque cavity through a tear on the fibrous cap was also demonstrated by injecting contrast agent (fig 2). Plaque rupture was also considered when an excavated plaque was found following extrusion of the atheromatous material (ulceration) (type II) (fig 3). In this case, no fibrous cap was visualised. In nonruptured plaques, the most stenotic segment was selected for analysis. Optimal images at end diastole were selected for analysis using the software built into the Hewlett-Packard Sonos intravascular machine.

The cross sectional area of a vessel was defined as the area inside the echo-dense perimeter of the adventitia, including lumen, plaque, and media. ${ }^{9}$ The lumen area was determined using planimetry of the ultrasound leading edge interface between lumen and

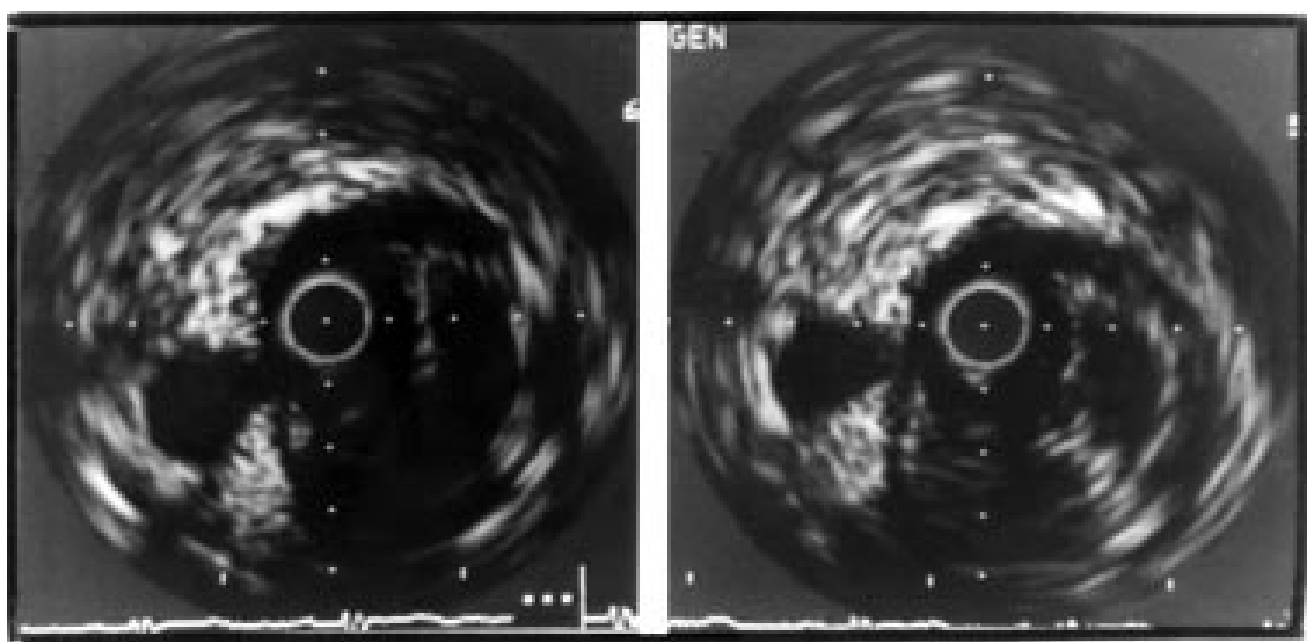

Figure 2 An IVUS image of plaque rupture. By injecting contrast material, a communication through the tear can be demonstrated (right). The lumen at 7 o'clock is the origin of a side branch. 


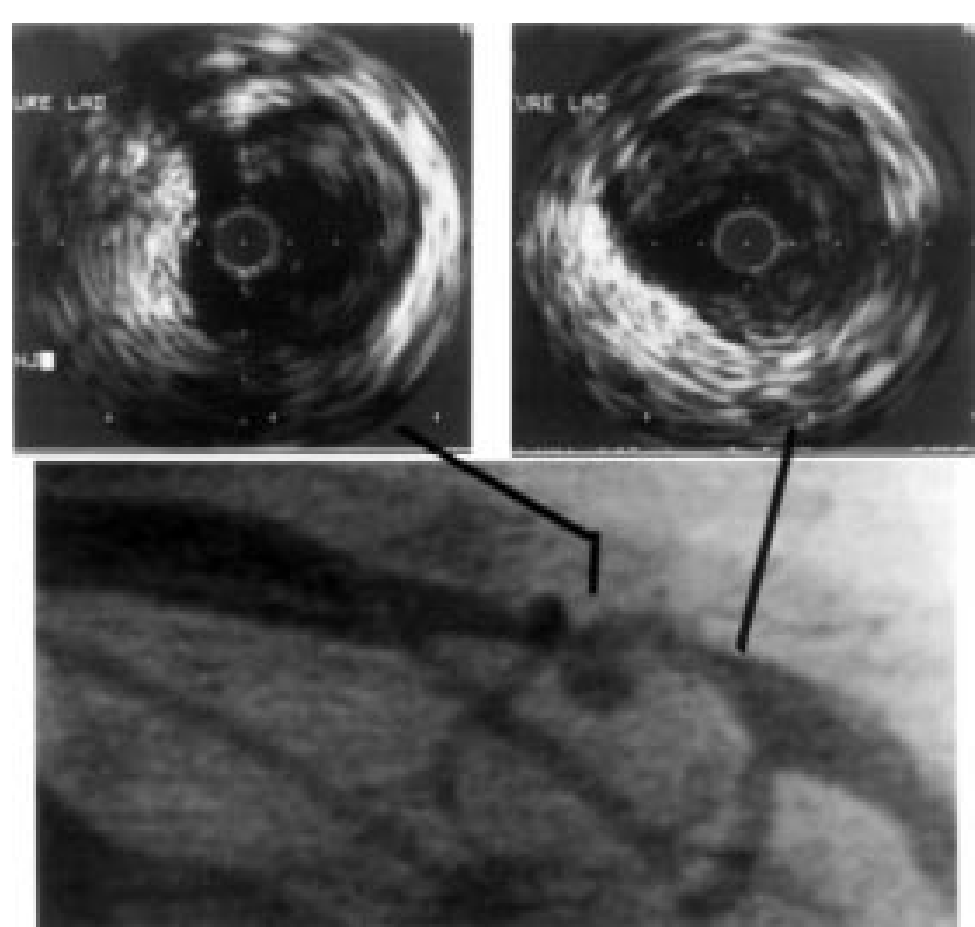

Figure 3 A patient with plaque ulceration, which presents as aneurysmal bulging on angiography. IVUS showed that the bulging is actually plaque ulceration (type II).

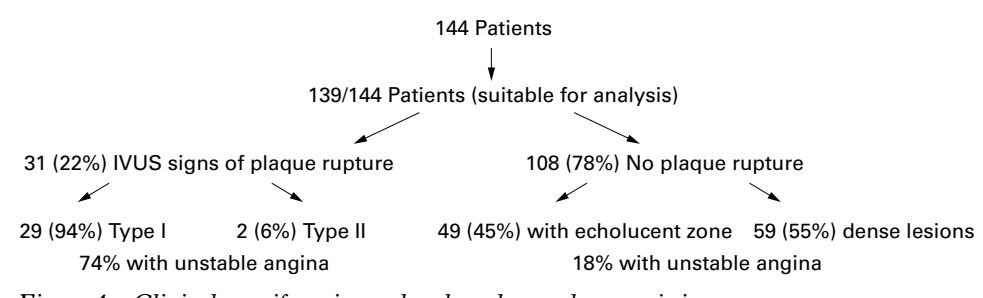

Figure 4 Clinical manifestations related to plaque characteristics.

plaque. The per cent area stenosis and per cent diameter stenosis were calculated using the lumen and vessel measurements from a single selected section. In ruptured plaques, the thickness of the fibrous cap, the tear size, the plaque size, and the size of emptied plaque cavity (echolucent, contrast medium filled area) were measured and the plaque cavity to plaque size ratio was calculated. The site (location) of the rupture in the plaque on the IVUS image was analysed according to a previous

Table 1 Clinical characteristics of the patients with (group A) and without (group B) plaque rupture

\begin{tabular}{llll}
\hline & Group $A$ & Group B & p Value \\
\hline Patients (n) & 31 & 108 & - \\
Sex (male) (mean (SD)) & $27(87 \%)$ & $92(85 \%)$ & NS \\
Age (years) & $55.0(8.5)$ & $57.7(9.3)$ & NS \\
Vessel & & & \\
$\quad$ LAD & 15 & 56 & - \\
LCX & 0 & 11 & - \\
RCA & 14 & 32 & - \\
LMCA & 1 & 6 & - \\
CABG & 1 & $49(45 \%)$ & NS \\
Hypertension & $19(61 \%)$ & $25(23 \%)$ & NS \\
Diabetes & $7(23 \%)$ & $62(57 \%)$ & NS \\
Nicotin & $21(68 \%)$ & $49(45 \%)$ & $<0.05$ \\
Hypercholesterolaemia & $23(74 \%)$ & $44(41 \%)$ & NS \\
Family history & $11(35 \%)$ & $38(35 \%)$ & NS \\
Obesity & $10(32 \%)$ & $19(18 \%)$ & $<0.001$ \\
Symptoms (UAP) & $23(74 \%)$ & & \\
\hline
\end{tabular}

$\star$ Total cholesterol $>5.2 \mathrm{mmol} / \mathrm{l}$.

CABG, coronary artery bypass graft; LAD, left anterior descending, LCX, left circumflex, LMCA, left main coronary artery; UAP, unstable angina pectoris. necropsy study. ${ }^{17}$ In non-ruptured plaques, we measured the echolucent zone size (indicating lipid core $)^{11}$; the thickness of the fibrous cap over the echolucent zone (distance from the lumen to the beginning of the echolucent zone); and the plaque size. The echolucent zone to plaque size ratio was then calculated.

The location of calcium deposits (superficial or deep) within the plaque was also evaluated. The severity of the calcification, according to the distribution and extent, was divided into the following five types: 0 , no calcium present; I, one quadrant $\leqslant 90^{\circ}$; II, two quadrant $\left(91^{\circ}\right.$ to $\left.180^{\circ}\right)$; III, three quadrant $\left(181^{\circ}\right.$ to $\left.270^{\circ}\right)$; IV, four quadrant $\left(271^{\circ}\right.$ to $\left.360^{\circ}\right) .^{18}$

Plaques are considered to be eccentric when the ratio of the thickest portion to the thinnest portion was more than 2.0.

CORONARY ANGIOGRAPHY AND ANGIOGRAM ANALYSIS

Siemens HICOR biplane catheterisation equipment was used for diagnostic coronary angiography (Siemens, Erlangen, Germany). All the angiograms are stored on laser disk. Coronary angiograms were assessed by two operators during coronary angiography and documented on hard copies. The angiographic characteristics were analysed according to the method described by Ambrose and colleagues ${ }^{7}$ : Concentric lesions - Symmetrical hourglass coronary artery narrowing; the borders are smooth or only slightly irregular.

Eccentric lesions-Eccentric narrowing with smooth (type I) or irregular (type II) borders. Multiple irregularities - Three or more serial closely spaced narrowings or severe diffuse irregularities within a vessel. ${ }^{7}$

Coronary aneurysm was defined when the maximum lumen diameter was 1.5 to 2 times larger than that of the adjacent proximal segment. $^{1920}$

STATISTICS

All the measurements and calculations were analysed using the WinStat (Statistics for Windows) and Primer statistic programs (McGraw-Hill). All the data are expressed as mean (SD). A Student's $t$ test analysis was used to assess the differences between the groups with and without plaque rupture. A p value $<0.05$ was considered statistically significant.

\section{Results}

CLINICAL CONSIDERATIONS

Of the 144 patients studied, the IVUS images were suitable for analysis in $139(97 \%)$. The patients were then divided into two groups (fig 4) according to whether or not a patient had echocardiographic signs of plaque rupture. Thirty one of the 139 patients (22\%; group A) were found to have plaque rupture (types I and II): 15 in the left anterior descending coronary artery, 14 in the right coronary artery, one in the left main coronary artery, and one in a left anterior descending artery bypass. No plaque ruptures were found in the other 108 patients (78\%; group B). The clinical details and characteristics of the two groups are listed in table 1. Unstable angina was present in 23 of the 31 

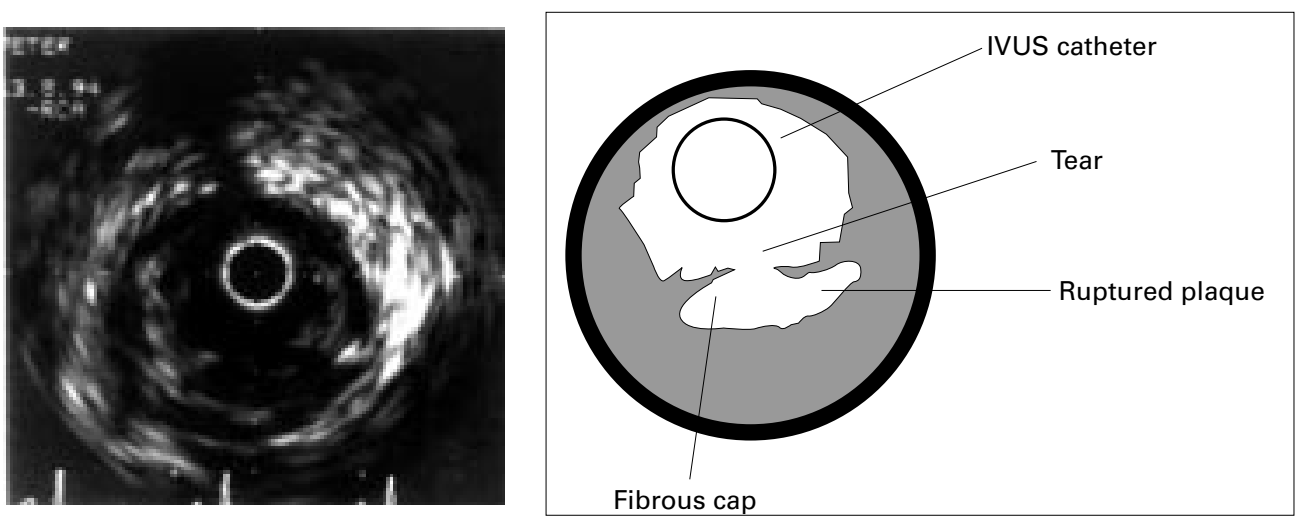

Figure 5 An eccentric plaque with a big tear, but the fibrous cap remains to be detected. Right panel is a schematic drawing of the cross section.

Table 2 Plaque dimensions and characteristics in the two groups

\begin{tabular}{llll}
\hline & Group $A$ & Group B & p Value \\
\hline Thickness of the fibrous cap (mm) & $0.47(0.20)(0.21$ to 0.76$)$ & $0.96(0.94)(0.4$ to 1.7$)$ & $<0.01$ \\
Tear size $(\mathrm{mm})$ & $0.83(0.29)$ & - & - \\
Eccentric & $94 \%$ & $64 \%$ & $<0.01$ \\
Plaque size $\left(\mathrm{mm}^{2}\right)$ & $11.7(7.0)(4.0$ to 30.1$)$ & $13.4(8.3)(4.0$ to 26.2$)$ & $\mathrm{NS}$ \\
Emptied plaque or lipid core size $\left(\mathrm{mm}^{2}\right)$ & $4.1(3.2)$ & $1.32(0.79)$ & $<0.001$ \\
Lipid to plaque ratio $(\%)$ & $38.5(17.1)$ & $11.2(8.9)$ & $<0.001$ \\
Stenosis (\%) & $56.2(16.5)$ & $67.9(13.4)$ & $<0.001$ \\
Superficial calcium deposits $(\mathrm{n}=29)^{\star}$ & $15(52 \%)$ & $54(51 \%)$ & $\mathrm{NS}$ \\
Deep calcium deposits & $5(17 \%)$ & $46(43 \%)$ & 0.019 \\
\hline
\end{tabular}

Values are mean (SD) and range.

$\star$ The two patients with plaque ulceration were excluded.

Group A, patients with plaque rupture; group B, patients without plaque rupture.

patients in group A (74\%), of whom two had had acute myocardial infarction within the previous two weeks. In contrast, only $19(18 \%)$ of the 108 patients presented with unstable angina in group $\mathrm{B}(\mathrm{p}<0.001)$. No differences were found between the two groups in relation to sex, age, and coronary risk factors except hypercholesterolaemia.

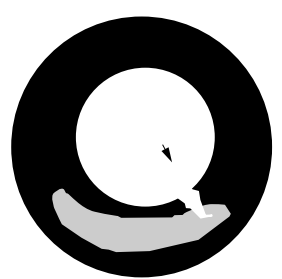

Pathology $49 \%$ IVUS

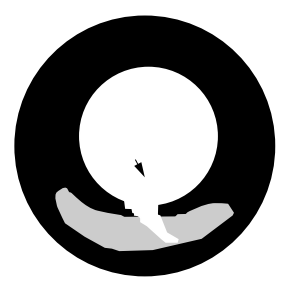

$29 \%$

$26 \%$

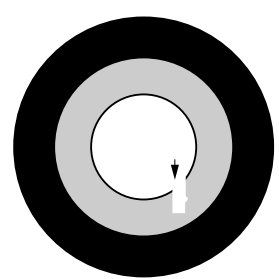

$5 \%$

$0 \%$

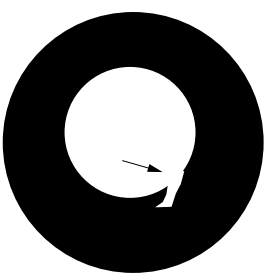

Pathology $13 \%$ IVUS $\quad 6 \%$

(10\% ulceration)

Figure 6 Localisation of the rupture in the plaques of the 31 patients in comparison with the pathological findings (modified from a schematic drawing by Richardson et al, ${ }^{17}$ with permission from Lancet). Arrows indicate the position of the tear on the plaque. The upper row indicates the pathological findings and the lower row the IVUS findings.
PLAQUE MORPHOLOGY

Ruptured plaques were characterised by a narrow tear in a thin fibrous cap with a deep echolucent zone within the plaque (figs 1 and 5, type I). Communication between excavated plaque and the lumen was demonstrated by injecting contrast agent (fig 2). Plaque ulcerations (type II) presented as a sac in the plaque, which was represented angiographically by a small aneurysmal bulge (fig 3). An echolucent zone was present in $49(45 \%)$ of the 108 patients without plaque rupture.

Data on calcium deposits (superficial or deep) and plaque morphology (eccentric and concentric) in the two groups are compared in table 2. No differences were found in relation to superficial calcium deposits. Deep calcium deposits, however, were more common in group B $(p=0.019)$. Although eccentric plaques appeared more often than concentric plaques in both groups ( $94 \% v 64 \%$ ), they were more common in ruptured plaques $(\mathrm{p}<0.01)$.

The localisation of the rupture is shown in fig 6 . The tear was frequently located at the lateral margin of the plaque $(55 \%)$. A central localisation was found in $26 \%$.

QUANTITATIVE ANALYSIS OF PLAQUES

The vessel and plaque dimensions in both groups are listed in table 2 . No significant differences between the two groups were found in relation to the vessel or plaque area. The mean (SD) per cent stenosis in patients with plaque rupture (56.2 (16.5)\%) was less than in patients without plaque rupture (67.9 $(13.4) \%)(p<0.001)$. The emptied plaque cavity area $\left(4.1(3.2) \mathrm{mm}^{2}\right)$ in patients with 

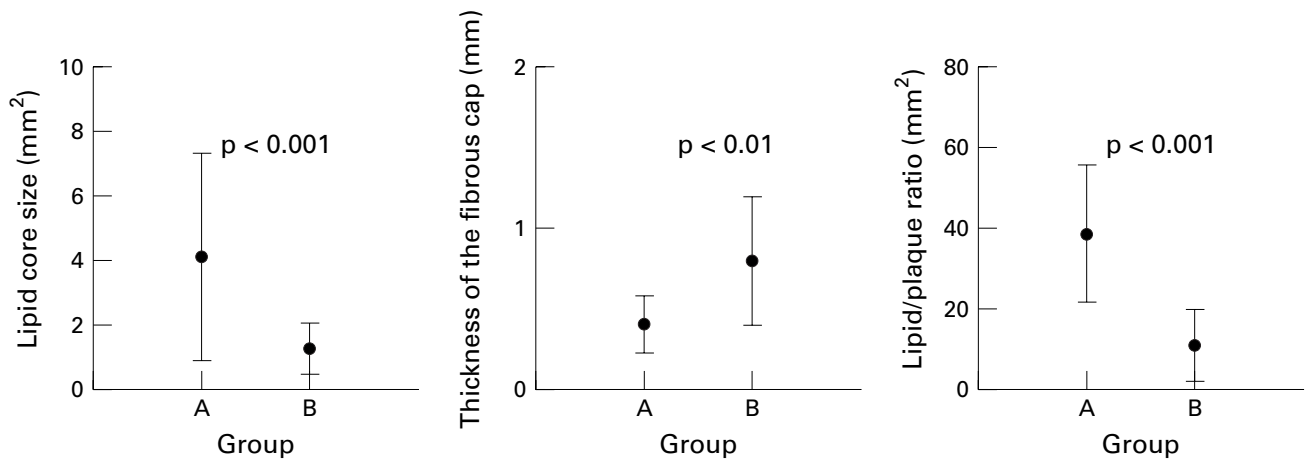

Figure 7 Comparison of echolucent zone size, lipid to plaque ratio, and the thickness of the fibrous cap between patients with plaque rupture (group $A$ ) and patients without plaque rupture (group B).

plaque rupture was larger than the echolucent zone $\left(1.32(0.79) \mathrm{mm}^{2}\right)$ in the $49(45 \%)$ of the 108 patients without plaque rupture $(\mathrm{p}<0.001)$ (fig 7). Similarly, the emptied plaque cavity to plaque size ratio in patients with plaque rupture (38.5 (17.1)\%) was larger than the echolucent zone to plaque ratio in patients without plaque rupture $(11.2(8.9) \%)$ $(\mathrm{p}<0.001$; fig 7$)$. The thickness of the fibrous cap in patients with plaque rupture $(0.47$ $(0.20) \mathrm{mm}$ ) was less than in patients without plaque rupture $(0.96(0.94) \mathrm{mm})(\mathrm{p}<0.001$; fig 7).

ANGIOGRAPHIC ASSESSMENT

Only $11(35 \%)$ of the 31 patients of group A had signs of an intimal flap or a contrast filled excavation angiographically (fig 3). Six patients $(19 \%)$ who were interpreted as having coronary aneurysms actually had emptied plaques. The other 14 patients (45\%) showed multiple irregularities or type II lesions. In group B, none showed angiographic aneurysmal dilatation or intimal flaps. Thirty seven $(34 \%)$ of the 108 patients had concentric lesions, 59 (55\%) had eccentric lesions, and the other 12 (11\%) had multiple irregularities.

\section{Discussion}

We found that plaque rupture of some form can be identified using IVUS. Ruptured plaques are characterised by an echolucent zone within a plaque, with a thin membrane representing a fibrous cap (type I), or by deep ulceration in the plaque (type II). Most ruptured plaques were eccentric lesions. Based on our results, the key indices related to plaque rupture seem to be lipid core size, the lipid to plaque size ratio, and the thickness of the fibrous cap. A lipid core larger than $1 \mathrm{~mm}^{2}$ or a lipid core to plaque ratio greater than $20 \%$ and a fibrous cap thinner than $0.7 \mathrm{~mm}$ correlated well with plaque rupture. Such plaques, if not already ruptured, may be called "plaques at risk" (vulnerable plaques). Similar observations are also reported in necropsy and atherectomy specimens where the lipid core that occupies a high proportion of overall plaque volume and a thin cap are indicators of the vulnerability of the plaque. ${ }^{21-24}$ However, the IVUS measurement is not exactly in accord with the pathological observations. ${ }^{21}$ Part of the discrepancy may reflect the IVUS technology currently available.
Coronary angiography, a contour technique that portrays only the silhouette of the contrast filled lumen with little information about the vessel wall, can detect only about one third of patients with plaque rupture. This indicates that the majority of the ruptured plaque would not be visible with this technique, for example the lesion in fig 2, when the fibrous cap is too thin.

No significant difference in relation to plaque size was found between groups A and B. These findings are in agreement with the pathological finding that plaque rupture depends on plaque type (composition) rather than on plaque size. ${ }^{12}{ }^{25}$ Nevertheless, a significant difference between the two groups was found for per cent stenosis. This might reflect plaque reorganisation during the long atherosclerotic process in group B. In patients with plaque rupture, the per cent luminal narrowing was less than in patients without plaque rupture. This correlates well with the theory that disruption of plaques occurs in nonstenotic arteries ${ }^{26} 27$ and endothelial denudation occurs in chronically stenotic arteries. ${ }^{28}$ Deep calcification was more common in group $\mathrm{B}$ than in group A. This finding accords with the pathological observation that stable plaques are more often calcified. ${ }^{26}$ In our study, only 31 of 139 patients $(22 \%)$ were found to have plaque rupture. However, in histopathological studies, plaque disruption has been reported in a ratio of 3 to 1 in one study ${ }^{28}$ and 1.3 to 1 in another. ${ }^{29}$ This discrepancy might be attributed to the selection process for specimens.

As early as 1926, Benson described thrombus formation after plaque rupture in coronary arteries. $^{30}$ Several pathologists have subsequently observed the same phenomenon. ${ }^{31-34}$ Initially, thrombus formation was misinterpreted as thrombus squeezed into the ruptured plaques. ${ }^{35}$ With IVUS, plaque rupture can be visualised in vivo. ${ }^{36}$ Recently, we demonstrated for the first time in vivo that plaque disruption reseals silently. ${ }^{15}$ This provides strong support for the hypothesis that plaque rupture and healing play an important role in the process of progression and regression of atherosclerosis. ${ }^{1-3}$ Not surprisingly, pathological studies have shown that there are layered thrombi overlying fissured plaques in most patients with unstable angina, leading to infarction or sudden death, and suggesting that most thrombi are formed 
by repeated mural deposits which progress to total vascular occlusion. ${ }^{1-3} 3738$ Serial morphological observations using intracoronary ultrasound may help to improve our knowledge of the mechanisms underlying the progression and regression of atherosclerosis, which is still poorly understood. On the other hand, the excavated material from the ruptured plaque may embolise peripherally as thrombotic or plaque material to be found in the distal microvascular coronary bed. ${ }^{39} 40$ Intermittent thrombus fragmentation may also result in peripheral embolisation (microemboli) with the same results. ${ }^{38} 40$ Since this process of plaque rupture and intermittent thrombus formation may occur silently - that is, without clinical symptoms-not all patients present with severe coronary syndromes. This is confirmed by our observation that eight patients with plaque rupture $(26 \%)$ presented with clinically stable angina. On the other hand, not all plaques with a lipid core show type I or II rupture characteristics when visualised by IVUS. This indicates these plaques may stay quiet for a while before rupturing. Part of the limitation may reflect the lack of resolution of the current ultrasound catheter systems, as mentioned above. Our data showed that a large percentage of patients with ruptured plaque have unstable angina, which strongly supports the conclusions derived from pathological observations.

THE SITE OF THE RUPTURE

Plaque rupture is affected by numerous factors, including increased shear stress injury, ${ }^{41}$ turbulent plaque injury, ${ }^{42}$ transient collapse of the stenosis, ${ }^{43}$ rupture of vasa vasorum, ${ }^{44}$ and circumferential stress within the plaque. ${ }^{17}{ }^{45}$ The site of rupture depends on the plaque morphology and its composition. ${ }^{45-47}$ Van der Wal et al reported that inflammation may destabilise the fibrous cap tissue and result in plaque rupture. ${ }^{48} \mathrm{~A}$ recent study showed that macrophages play a significant role in plaque rupture because they may release lytic enzymes that degrade the fibrous cap, causing the atherosclerotic plaques to rupture. ${ }^{23}$ Our findings are in agreement with the observations of Richardson et al that the tears frequently occurred at the lateral margin of the plaque (fig 6) ${ }^{17}$ There are discrepancies relating to superficial tears and ulceration. IVUS data in the present study showed a lower percentage of superficial tears than pathological examination, detecting plaque ulceration in $10 \%$ of the patients. This may reflect the resolution of current intravascular ultrasound, which is in the range of 150 to $200 \mu \mathrm{m}$.

ROLE OF PLAQUE RUPTURE IN THE PROCESS OF PLAQUE PROGRESSION

Following plaque rupture blood enters the emptied plaque and the exposed subendothelial tissue triggers the thrombotic process described above. Exposure of subendothelial tissue may result in platelet accumulation, which in turn may result in release of platelet derived growth factor and lead to smooth muscle cell proliferation. ${ }^{49}$ The latter may account for progression of atherosclerosis. If the host wins in this battle, then in several episodes the plaques stabilise and there may be calcium deposits or fibrosis. ${ }^{41}$ If the coagulation system wins, with or without the help of vascular spasm in that segment, then clinically acute coronary syndromes result. ${ }^{38}{ }^{40}$ The observation in our study that per cent stenosis in patients with stable plaques was greater than in patients with unstable plaques may also be in agreement with this hypothesis.

\section{LIMITATIONS OF THE STUDY}

We visualised only the main epicardial coronary arteries and not the side branches, and thus may have underestimated the true incidence of plaque rupture. Nevertheless, coronary atherosclerosis most often involves the proximal portion of the coronary arteries, usually opposite the branches. ${ }^{50}$ Thus the underestimation seems likely to be insignificant. With IVUS, it may be difficult to identify a ruptured plaque when the rupture is covered or filled by echoreflectant mural thrombi. The events after plaque rupture may be followed by total or subtotal vessel occlusion by thrombus formation. Plaque rupture may also be followed by plaque reorganisation, as mentioned above. This situation may be detected by the indirect sign of a layered appearance over the plaque. ${ }^{34}$ Preliminary studies on tissue characterisation in vitro have shown that thrombi result in clearly different backscatter signals in comparison with fibrous tissue and lipid accumulation. This will enable us to differentiate different types of tissue in the future.

\section{CLINICAL IMPLICATIONS}

Plaque rupture is the key event leading to acute coronary syndromes. Identification of plaque rupture is therefore very important in patient management and for improving prognosis. For the first time, the anatomical pathology and natural history of plaque rupture can be studied in vivo. The future studies should aim at the identification of the "plaque at risk" (vulnerable plaque) before it ruptures and provide morphological evidence of the development of coronary syndromes as well.

1 Davies MJ, Thomas AC. Plaque fissuring-the cause of acute myocardial infarction, sudden ischemic death, and crescendo angina. Br Heart $\mathcal{F}$ 1985;53:363-73.

2 Davies MJ, Bland MJ, Hangartner WR, et al. Factors influencing the presence or absence of acute coronary thrombi in sudden ischemic death. Eur Heart $\mathcal{F}$ 1989;10:203-8.

3 Fuster V, Stein B, Ambrose JA, et al. Atherosclerotic plaque Fuster V, Stein B, Ambrose JA, et al. Atherosclerotic plaque
rupture and thrombosis: evolving concepts. Circulation 1990;82 (suppl II):47-59.

4 Ridolfi RL, Hutchins GM. The relationship between coronary artery lesions and myocardial infarcts: ulceration of atherosclerotic plaques precipitating coronary thrombosis. Am Heart f 1977;93:468-86.

5 Fuster V, Badimon L, Badimon JJ, et al. The pathogenesis of coronary disease and the acute coronary syndromes. $N$ Engl f Med 1992;326:242-318.

6 Erbel R, Ge J, Görge G, et al. Intravaskuläre Sonographie bei koronarer Herzkrankheit, Neue Aspekte zur Pathogenese. Dtsch Med Wochenschr 1995;120:847-54.

7 Ambrose JA, Winters SL, Stern A, et al. Angiographic morphology and the pathogenesis of unstable angina pectoris. $f$ Am Coll Cardiol 1985;5:609-16.

8 MacIsaac AI, Thomas JD, Topol EJ. Toward the quiescent coronary plaque. 7 Am Coll Cardiol 1993;22:1228-41.

9 Ge J, Erbel R, Gerber T, et al. Intravascular ultrasound imaging of angiographically normal coronary arteries: a imaging of angiographically normal coronary arteri
prospective study in vivo. Br Heart $\mathcal{F}$ 1994;71:572-8.

10 Gr J, Erbel R, Zamorano J, et al. Coronary artery remodeling in atherosclerotic disease: an intravascular ultrasound study in vivo. Coron Artery Dis 1993;4:981-6. 
11 Di Mario C, The SHK, Madretsma S, et al. Detection and characterization of vascular lesions by intravascular
ultrasound: an in vitro study correlated with histology. $\mathcal{F}$ Am Soc Echocardiogr 1992;5:135-46.

12 Tobis JM, Mallery J, Mahon D, et al. Intravascular ultrasound imaging of human coronary arteries in vivo. Circulation 1991;83:913-26.

13 Fitzgerald PJ, Ports TA, Yock PY. Contribution of localized calcium deposits to dissection after angioplasty, an observational study using intravascular ultrasound. Circulation 1992;86:64-70

14 Honye J, Mahon DJ, Jain A, et al. Morphological effects of coronary balloon angioplasty in vivo assessed by intravascular ultrasound imaging. Circulation 1992;85:1012-25.

15 Ge J, Haude M, Görge G, et al. Silent healing of spontaneous plaque rupture demonstrated by intracoronary ultrasound. Eur Heart f 1995;16:1149-51.

16 Braunwald E. Unstable angina, a classification. Circulation 1989;80:410-14.

17 Richardson PD, Davies MJ, Born GVR. Influence of plaque configuration and stress distribution on fissuring of coronconfiguration and stress distribution on fissuring

18 Mintz GS, Popma JJ, Pichard AD, et al. Patterns of calcification in coronary artery disease. A statistical analysis of intravascular ultrasound and coronary angiography in 1155 lesions. Circulation 1995;91:1959-65.

19 Befeler A, Aranda JM, Embi A, et al. Coronary artery aneurysms: study of their etiology, clinical cause and effect on left ventricular function and prognosis. Am f Med 1977; 62:597-607.

$20 \mathrm{Ge}$ J, Liu F, Kearney P, et al. Intravascular ultrasound approach to the diagnosis of coronary aneurysms. An Heart f 1995;130:765-71.

21 Davies M, Richardson P, Woolf N, et al. Risk of thrombosis in human atherosclerotic plaques: role of extracellular lipid, macrophage, and smooth muscle cell content. Br Heart $\mathcal{F}$ 1993;69:377-81.

22 Falk E. Morphological features of unstable atherosclerotic plaques underlying acute coronary syndromes. $A m$ F plaques underlying acute

23 Moreno P, Falk E, Palacios IF, et al. Macrophage infiltration in acute coronary syndromes: implications for plaque rupture. Circulation 1995;90:775-8.

24 Annex B, Denning S, Channon K, et al. Differential expression of tissue factor protein in directional atherectomy specimens from patients with stable and unstable coronary syndromes. Circulation 1995;91:619-22.

25 Falk E. Why do plaques rupture? Circulation 1992; 86(suppl):30-42.

26 Stary HC, Chandler AB, Dinsmore RE, et al. A definition of advanced types of atherosclerotic lesions and a histological classification of atherosclerosis: a report from the Committee on Vascular Lesions of the Council on Atherosclerosis, American Heart Association. Circulation 1995;92: 1355-74.

27 Baumgart D, Liu F, Haude $M$, et al. Acute plaque rupture and myocardial stunning in patient with normal coronary and myocardial stunning in patient with

28 Davies MJ. A macro and micro view of coronary vascular insult in ischemic heart disease. Circulation 1990;82(supp I): $1138-46$.

29 Farb A, Burke AP, Tang AL, et al. Coronary plaque erosion without rupture into a lipid core: a frequent cause of coronary thrombosis in sudden coronary death. Circulation 1996;93:1354-63.

30 Benson RL. The present status of coronary arterial disease. Arch Pathol 1926;2:870-916.

31 Koch W, Kong LC. Über die Formen des Coronarverschlusses. Die Anderung im Coronarkreislauf und die Beziehung zur Angina pectoris. Beitr Pathol Anat 1932:90:21-84
32 Leary T. Experimental atherosclerosis in rabbit compared with human (coronary) atherosclerosis. Arch Pathol 1934; 17:453-92.

33 Clark E, Graef I, Chasis H. Thrombosis of aorta and coronary arteries, with special reference to "fibrinoid" lesions. Arch Pathol 1936;22:183-212.

34 Kearney P, Erbel R, Rupprecht HJ, et al. Differences in the morphology of unstable and stable coronary lesions and their impact on the mechanisms of angioplasty. An in vivo study with intravascular ultrasound. Eur Heart F 1996;17: 721-30.

35 Sinapius D. Relationship between coronary artery thrombosis and myocardial infarction. Dtsch Med Wochenschr 1972; 97:443-8.

36 Zamorano J, Erbel R, Ge J, et al. Spontaneous plaque rupture visualized by intravascular ultrasound. Eur Heart $f$ 1994;15:131-3.

37 Falk E. Plaque rupture with severe pre-existing stenosis precipitating coronary thrombosis: characteristics of coronary atherosclerotic plaques underlying fatal occlusive thrombi. Br Heart f 1983;50:127-34.

38 Falk E. Unstable angina with fatal outcome: dynamic coronary thrombosis leading to infarction and/or sudden death: autopsy evidence of recurrent mural thrombosis with peripheral embolization culminating in total vascular occlusion. Circulation 1985;71:699-708.

39 Hort W. Der arteriosklerotische Polsterriß. Versicherungsmedizin 1991;43:151-4.

40 Jorgensen L, Rowsell HC, Hovig $\mathrm{T}$, et al. Adenosine diphosphate-induced platelet aggregation and myocardial infarction in swine. Lab Invest 1967;17:616-44.

41 Gertz SD, Roberts WC. Hemodynamic shear stress force in rupture of coronary arterial atherosclerotic plaques. Am $\mathcal{F}$ Cardiol 1990;66:1368-72.

42 Loree HM, Kamm RD, Atkinson CM, et al. Turbulent pressure fluctuations on surface of model vascular stenosis. Am f Physiol 1991;261:H644-50.

43 Binns RL, Ku DN. Effect of stenosis on wall motion: a possible mechanism of stroke and transient ischemic attack. Arteriosclerosis 1989;9:842-7.

44 Barger AC, Beeuwkes R, Lainey LL, et al. Hypothesis: vasa vasorum and neovascularization of human coronary arteries: a possible role in the pathophysiology of atherosclerosis. N Engl f Med 1991;88:8154-8.

45 Loree HM, Kamm RD, Stringfellow RG, et al. Effects of fibrous cap thickness on peak circumferential stress in model atherosclerotic vessels. Circ Res 1992;71:850-8.

46 Fernandez OA, Badimon JJ, Falk E, et al. Characterization of the relative thrombogenicity of atherosclerotic plaque components: implications for consequences of plaque rupture. 7 Am Coll Cardiol 1994;23:1562-9.

47 Ge J, Liu F, Görge G, et al. Visualization of ulcerated unstable plaques by intracoronary ultrasound. $Z$ Kardiol 1995;84:I-86.

48 van der Wal AC, Becker AE, van der Loos CM, et al. Site of intimal rupture or erosion of thrombosed coronary atherosclerotic plaques is characterized by an inflammatory process irrespective of the dominant plaque morphology. Circulation 1994;89:36-44.

49 Wilentz JR, Sanborn TA, Haudenschild CC, et al. Platelet accumulation in experimental angioplasty: time course and relation to vascular injury. Circulation 1987;75:63642

50 Montenegro MR, Eggen DA. Topography of atherosclerosis in the coronary arteries. Lab Invest 1968;18:586-93. 